\title{
Review
}

\section{Assessing Drug-Induced Dyskinesia in the Clinic, the Laboratory and the Natural Environment of Patients}

\author{
Benoit Carignan $^{\mathrm{a}}$, Jean-François Daneault ${ }^{\mathrm{b}}$ and Christian Duval ${ }^{\mathrm{c}, *}$ \\ a Département des Sciences Biologiques, Université du Québec à Montréal, Montréal, QC, Canada \\ ${ }^{\mathrm{b}}$ Department of Neurology and Neurosurgery, Montreal Neurological Institute, McGill University, Montreal, \\ QC, Canada \\ ${ }^{\mathrm{c}}$ Département de Kinanthropologie, Université du Québec à Montréal, Montréal, QC, Canada
}

\begin{abstract}
The assessment of drug-induced dyskinesia (DID) in Parkinson's disease represents a formidable challenge for clinicians and researchers alike. The present review describes the current assessment tools used in the clinic, where different scales have been developed for monitoring levels of DID in patients. We also review laboratory tools used to assess the quantity and characteristics of DID. Finally, we review assessment methods currently in development for monitoring DID and voluntary mobility in the natural living environment of patients. Here, we discuss the strengths and weaknesses of these tools as it pertains to their efficacy in assessing the quantity of DID, its characteristics, as well as its impact on the quality of life of patients. Finally, we discuss ongoing challenges and research questions that may guide future development of assessment methods aimed at monitoring DID and its impact on daily lives of patients.
\end{abstract}

Keywords: Dyskinesia, LID, chorea, motion tracking, questionnaire, Parkinson, scale, assessment, treatment, involuntary movement

\section{INTRODUCTION}

Levodopa-induced dyskinesia (LID) represents the main side effect of long-term treatment in Parkinson's disease (PD). LID is a type of motor fluctuation which can stem from poor management of exogenic levodopa by remaining dopamine neurons, as well as changes in the sensitivity of post-synaptic dopamine receptors [1-3]. While LID is a commonly used term in the literature to describe motor fluctuations due to PD

\footnotetext{
*Correspondence to: Christian Duval, Département de Kinanthropologie, Université du Québec à Montréal, C.P. 8888, Succursale Centre-Ville, Montréal (Québec), Canada, H3C 3P8, Bureau \#: SB4420. Tel.: (514) 9873000; Ext: 4440; Fax: (514) 987 6616; Courriel: duval.christian@uqam.ca.
}

medication, drug-induced dyskinesia (DID) will be utilized in this review as it includes other possible causes of motor fluctuations seen in PD. Indeed, these motor fluctuations can also be induced by dopamine agonists $[4,5]$ possibly thru changes in globus pallidus internus (GPi) neuronal activity, such as firing rate and firing frequency, mediated by $\mathrm{D}_{1}$ and $\mathrm{D}_{2}$ dopamine receptors [5]. DID can also stem from the addition of COMT-I agents to the treatment regimen [6]; which block the peripheral metabolism of levodopa to 3-0-methyldopa (3-OMD) [7], and also slightly prolonging elimination half-life of levodopa [8] resulting in increased bioavailability of levodopa and a measurable increase in the amount of levodopa entering the brain. The resulting motor fluctuation is hyperkinesia 
manifesting as uncontrolled limb movements that can be present throughout the body. While there are several classifications or types of DID, such as dystonic, ballism and myoclonus [9-12], the most common type is choreic peak-dose dyskinesia which is random in appearance, involuntary, purposeless, non-rhythmic, abrupt, rapid, irregular, and unsustained [9]. This type of DID produces unpredictable movements of the limbs while being temporally well time locked to the medication intake in most instances. Proper assessment of the characteristics of DID in PD is essential in order to monitor the effect of treatment, as well as how DID influences voluntary motor acts of patients. This latter issue is important since it ultimately provides information as to when DID has an impact on the quality of life of patients. However, the assessment of DID, and its impact on the daily lives of patients, has always been a formidable challenge to clinicians and researchers alike. In this review, we will present an overview of the current methods of assessing DID in the clinic, in the laboratory, as well as in the natural living environment of patients. For each of these sections, we will discuss strengths and weaknesses of these assessment methods. Finally, we will discuss future challenges related to the proper quantification and characterization of DID. The methods described here will only cover efforts to assess DID in PD, while recognizing that some methods originated from assessing other types of motor dysfunctions such as chorea in Huntington's disease, tardive dyskinesia, etc.

\section{CLINICAL ASSESSMENT OF DID}

The fast pace of current movement disorders clinics requires effective means of assessing patients so as to keep track of changes associated with disease course. This is the basis for the development of clinical scales. Accordingly, the main goal of a clinical assessment should be to efficiently provide a clinical picture of the state of a patient at one point in time. As well, clinical scales should be geared towards assessing the impact of motor symptoms on the daily lives of patients. The assessor (usually a clinician or trained medical personnel) will simply enter their assessment of the level of DID on a scale or questionnaire. In some cases, the patient may enter the required information on a form. The origins of these assessment scales for DID can be divided into three main groups. The first group is comprised of scales examining global motor disabilities in PD, in which elements target DID assessment. This is the case for the Unified Parkinson's disease rating Scale (UPDRS) [13] and now the Movement Disorders Society sponsored revision of the UPDRS (MDS-UPDRS) [14], or the Core Assessment Protocol for Intracerebral Transplantation (CAPIT) [15], in which sub-scores are specifically targeting the level of DID. The second group of scales was developed based on the assessment of involuntary movements associated with other syndromes, such as the Abnormal Involuntary Movement Scale (AIMS) [16]. The AIMS was first developed for tardive dyskinesia, and was subsequently used for Huntington's chorea and PD DID. Finally, the third group was developed specifically to assess dyskinesia, such as the Unified Dyskinesia Rating Scale (UDysRS) [17], the Rush Dyskinesia Rating Scale [18], and the Clinical Dyskinesia Rating Scale (CDRS) [19]. Other scales were specifically developed to assess the impact of DID on the daily life of patients. The Lang-Fahn Activities of Daily Living Dyskinesia Scale [20] and the Parkinson's disease Dyskinesia Scale (PDYS-26) [21] are examples of those types of scales. An exhaustive evaluation of each of these scales was performed by the task force on scales to assess dyskinesia [22]. In brief, they view as an optimal scale one that should be able to capture the level of impairment and level of disability, the anatomical location of DID, changes over time and finally, the patient's own assessment of their DID. While they recommend the use of the AIMS, as well as the Rush Dyskinesia Rating Scale based on their evaluation criteria, despite each having some shortcomings, all the other reviewed scales were classified as being suggested for use since they each partially fulfilled their assessment criterion. They point out that scales being only suggested for use were also validated, reliable and sensitive enough for the assessment of dyskinesia. Each of these scales has their own advantages, such as providing quick assessment, as well as good assessment of DID location on the body (e.g. the AIMS) and the impact of DID on the daily lives of patients (e.g. the PDYS-26) while each having limitations. For instance, the mere nature of the clinical settings in which clinicians and medical personnel work, i.e. space and time constraints, requires that the assessment be performed simply and quickly. Limiting the efficacy of such evaluation is the fact that DID is inherently variable during the day within a given patient [23]. Furthermore, we recently demonstrated that the amplitude and location of dyskinetic movements change even over short periods of times [24]. We also demonstrated that the amplitude of dyskinesia was highly sensitive to the voluntary movements made in other parts of the body [24]. These issues make it even more difficult to capture the 
clinical picture of DID in a patient, let alone determine how it will influence their daily lives. Nonetheless, there are clinical scales that incorporate self-reported evaluation by the patient, such as the UDysRS, which can provide a better picture of the fluctuations in DID over the preceding week. Indeed, to our knowledge, the UDysRS is the only clinical scale for which temporal stability has been established [25]. This indicates that as long as the evaluation is performed when the patient is in a known stable medication state (i.e. ON or OFF), one evaluation with the UDysRS is a reliable estimate of the score that would be obtained at any other time for that patient. One more limitation in the assessment of DID is the difficulty to distinguish, in some cases, between end-dose dystonia and choreic-type DID.

All of the aforementioned scales were validated on the basis that the evaluator can differentiate dyskinesia from other forms of involuntary movements. Their intra- and inter-rater reliability has also been verified and confirmed by many studies. They were demonstrated as being sensitive, as long as the evaluator is sufficiently trained and can detect the involuntary movements when present. However, it is the resolution of some items in certain scales that may prove problematic, especially when they are used to assess the effect of treatment. Indeed, several clinical scales use items that can be scored between 0 and 4 (e.g. UDysRS and MDS-UPDRS) or 1 to 5 (e.g. AIMS). Other scales, while still providing scores between 0 and 4 , allow for possible intervals of 0.5 (for example, the UPDRS). This means that each of those items has a maximal resolution of approximately 3 bits (i.e. 9 increments in scoring), which is hardly enough to detect subtle changes in DID state over time or as a result of treatment. Of course, when adding all the items of a particular scale, such as in the AIMS, a better resolution of whole-body dyskinesia can be obtained. While clinical assessment may provide information as to the location and severity of DID, subtle changes in amplitude, relative distribution within each limb and other characteristics such as velocity and frequency cannot be evaluated using these methods. Below, we will make the case as to why tests with a higher resolution may be important. Currently, however, clinical scales represent the best tool available to clinicians for quick, reliable and affordable assessment of the clinical state of the patients as it pertains to DID. Most of these scales are readily available as they can be downloaded from the web (for example see the Movement Disorders Society website [26]). It is also the most practical mean of assessing DID in large cohorts as they are inexpensive and require relatively little training for their use and interpretation of results.

\section{LABORATORY ASSESSMENT OF DID}

In order to better understand the impact of DID on patients' motor performance, as well as to investigate the pathophysiology of those clinical manifestations; one must use higher-resolution instruments that provide ways to capture the actual movement to be examined using more sophisticated analytical methods. The goal of these tools should be to provide more accurate quantification of the characteristics of DID, such as the magnitude, velocity, localization and any other pertinent property of DID relating to a specific question. Several methods have been used to assess DID in patients with PD, such as surface electromyography (sEMG), wearable sensors, camerabased and magnetic motion tracking systems. For instance, Yanagisawa and Nezu [27] used sEMG to study DID, and found irregular bursts in the EMG pattern. This method proved efficient in assessing the irregular nature of the interplay between agonists and antagonists muscles during DID. Burkhard [28] developed a sensor that measured rotational movements using a double-ended quartz tuning fork driven to oscillate at $10 \mathrm{kHz}$. This piezoelectric device measured torque of the examined limb during DID. Mounted on both hands, the device was able to detect pronationsupination and flexion extension of the wrist which helped in assessing the magnitude of DID during quiet stance while comparing both sides of the body. Their device correlated well with clinical ratings of DID severity using a customized rating scale. They also provided the main frequencies of DID, which lie somewhere around $3.5 \mathrm{~Hz}$ (from the velocity power spectrum). This frequency of DID has been confirmed by us [24, 29, 30] and others [31-33] using other laboratory assessment methods. Liu et al. [31] developed a way to assess DID of the arm using digitised spiraldrawing tasks. Using spectral analysis of the drawings, they were able to identify dyskinesia, voluntary movements and tremor. Their assessment of DID using this task correlated with clinical assessments using the UPDRS and the Bain Dyskinesia Scale. This method of assessing DID has been useful to assess the effect of different treatments on DID [34]. Rao et al. [35] have developed video-based assessment methods by which the level of DID can be assessed using clinical video recordings of patients. This method called for the identification of landmarks on the patients (limited to the head and shoulder in this study) that could be tracked during multiple frames using a custom-designed algorithm. They also found a high correlation between their evaluation system and the assessment performed by a 
trained clinician using the UPDRS. The advantage of this system is that it can be used on previously recorded evaluations; however it is time consuming and is currently limited in the number of landmarks which can be assessed. Chung et al. [36] have proposed the use of force platforms to assess the magnitude of DID. They simply used the amount of center of pressure displacement to calculate the level of DID. They found a high correlation between the area under the curve, i.e. the magnitude of center of pressure movement, and the clinical evaluation of DID using the AIMS.

These aforementioned methods have some limitations such as providing limited information about the location of the DID, as well as the type of dyskinesia present in that patient; i.e. choreic or dystonic. Since DID can be present throughout the body, it would be logical to attempt to capture the entire body in order to assess its characteristics. Current video-based systems (often called optical systems) using passive reflective markers or active infrared-emitting markers are ubiquitous in biomechanics laboratories around the world. They are highly accurate, and provide translational data that can be used for complete analysis of movement. Since the patient is not wired to a computer in any way, the field of recording can be customized. However, the unpredictable nature of DID renders the positioning of cameras and markers on the body extremely difficult. In fact, the unpredictability of motion may cause some of those rigid markers to be hidden during recording. Accordingly, the line-of-sight issue represents a formidable challenge. Another aspect that hinders the use of optical systems is the fact that rigid bodies (i.e. multiple markers) must be placed on each limb to capture longitudinal rotations of limbs, so as to provide full 6 degrees of freedom data for each limb in displacement ( $\mathrm{x}, \mathrm{y}$ and $\mathrm{z}$ in 3D space), as well as Euler angles (pitch, roll and yaw). This increases the number of markers to be tracked, raising the probability of losing sight of some of those markers. This is probably one of the reasons why, to our knowledge, no attempt has been made to quantify whole-body DID with such systems. However, these types of systems have been used to assess DID in specific limbs, providing highly accurate information on the characteristics of DID within the examined limb [37]. A type of system that can circumvent the line of sight issue is a magnetic tracker system. Here, a box containing three coils mounted orthogonally generates consecutive electro-magnetic pulses that are detected by multiple sensors placed strategically on the body. Each sensor can provide data of the 6 degrees of freedom which allows, for example, the patient to be sitting down in front of a table, while performing motor tasks. Using such a system, we were able to demonstrate the influence of DID on voluntary movements and the influence of voluntary movements on DID [38, 39]. The same relationship was demonstrated in Huntington disease chorea [24, 40]. In addition, we were able to describe the variability of DID in time and space (i.e. the location of DID throughout the body) [24], as well as provide information about the movement patterns of DID [30, 41, 42]. We were also able to compare the dynamic relationship between voluntary movements and DID in PD and Huntington disease chorea [29]. One drawback of this type of equipment is that it requires time to setup the sensors and to assign them to specific center of mass of the limbs on which they are to be installed. This type of equipment is also very sensitive to magnetic disturbances which require careful planning and mapping prior to installation.

While the tools described above are readily available, very few groups have taken up the challenge of assessing and characterizing DID in a laboratory setting. As for the clinical assessment, the issue of DID variability [23] must be once again addressed. While laboratory assessment may provide a better resolution of DID characteristics, it can only do so on a limited time scale. Indeed, for most research on DID, it is not feasible to bring patients within a laboratory setting for several hours and expect them to display "normal" DID. Another issue is that differentiating voluntary movements from involuntary movements is problematic. In fact, several groups have worked on differentiating voluntary movements from DID (see next section for details). Tremor is another type of involuntary movement that must be differentiated from DID in order to adequately interpret the results. While tremor and DID have different intrinsic properties, time- and frequency-domain analyses must be performed in order to differentiate both types of movements. Finally, most of these equipments require substantial financial expenses as well as relatively long and arduous training for their use, but also for the analysis and interpretation of the results.

\section{NATURAL LIVING ENVIRONMENT ASSESSMENT OF DID}

One important aspect that is not well understood about DID is how it affects the lives of patients in their natural environment. Current clinical scales as well as laboratory assessments for DID will provide some information about the effect of treatments on the 
everyday life of patients, but rely on measures of impairment made in the clinic, sometimes on measures of disability, but rarely on measures of handicap. Clinicians must then rely on feedback from patients, which can be difficult to interpret. For instance, the patient may report benefits from a change of medication, but the spouse may contradict this claim by telling the treating physician that the overall amount of mobility is reduced (e.g. increased apathy). These problems represent the driving force behind the developments of new techniques for the monitoring of DID in the natural environment of patients. To date, attempts made by clinical scales and laboratory assessments to capture the effect of DID on the daily activities of patients represent valid efforts. However, they do not capture the effect of DID on the entire repertoire of movements of the patient during their daily routine, which limits our ability to determine when DID is detrimental to them, and when it is not. Then, the objective should be to develop nonintrusive systems that can monitor not only the quantity of mobility, but also provide means to assess its quality.

In recent years, there has been tremendous effort in developing technological methods to assess DID in an ecological manner, meaning that sensors would be worn by patients with minimal influence of the device itself, and the experimentalist. Some have used accelerometers, gyroscopes or combinations of those methods and others called inertial motion units (IMU). The advantages of using such systems are numerous. First, it allows for the assessment of movement in the natural living environment of patients, with minimal interference by the experimentalist. This is important since no optical system, to date, will have the flexibility provided by wearable accelerometers or IMU in a home setting. Second, it may be used for longterm recordings (i.e. several hours), which provides a more accurate representation of changes in motor symptomatology throughout the time course of treatment, i.e. the pharmacodynamics. Finally, they may help us eventually discern when DID become a major burden for patients by determining, for instance, when compensatory strategies used by patients no longer work. Indeed, the majority of these equipments, similar to those that are used in the laboratory, can capture enough biomechanical information to develop analytical models to assess these and other relevant features.

Manson et al. [33] used a sensor composed of three orthogonally-mounted uniaxial piezoelectric accelerometers. This sensor was mounted on the shoulder of a patient. The patient also wore a data logger on his belt with a power supply which allowed for autonomy of 15 hours. Patients were asked to perform motor tasks that were included in modified clinical scales such as the AIMS and the Rush Dyskinesia Scale. They found that levels of acceleration were well correlated with clinical scores. One issue encountered was that the voluntary movements overlapped with the involuntary movements, even though dyskinetic patients showed higher peaks of acceleration between 1 and $3 \mathrm{~Hz}$. The issue of differentiating involuntary from voluntary movements is a complex matter that is addressed below.

Hoff et al. [43] recognized the need to assess more than only one limb in order to have a clearer clinical picture of DID. They installed eight accelerometers mounted in pairs to cover the coronal and sagittal plane of the upper leg, wrist, truck and upper arm. The severity of DID was measured against a modified AIMS, and CDRS. Their assessment method proved to be effective when patients refrained from performing voluntary movements, but less effective during voluntary motor tasks. They tackled this issue by examining whether assessing acceleration of multiple limbs could help in detecting $\mathrm{ON}$ and OFF clinical states during a 24 hour recording [44]. They determined that their assessment method was not sensitive enough for automated ON-OFF detection in individual patients. They recognized that voluntary movements again overlapped with involuntary movements, rendering clinical assessment of either bradykinesia, or dyskinesia more difficult. Indeed, a voluntary self-restriction in motor activities may result in a wrong OFF detection. Keijsers et al. [45], who took the data collected in the aforementioned study of Hoff et al. [43], circumvented these issues by applying neural network analysis instead of simpler linear discriminate analysis. They were able to better distinguish voluntary from involuntary movements, but this evaluation was still not optimal. Keijsers et al. [46] later tested improved neural network algorithms on data collected in a home setting while patients performed pre-determined everyday activities for 2.5 hours. This time, they used six sites to place the accelerometers: both upper arms, both upper legs, wrist of the most dyskinetic side, and trunk. Several variables were fed into a multilayer perceptron (feedforward artificial neural network model) made of one input layer, one hidden layer and one output layer. Results showed that the neural network was able to detect and assess the magnitude of DID, as well as discriminate voluntary movements from involuntary ones. With this study, the group of Keijsers demonstrated that neural networks were efficient in detecting interactions between movement variables and determined 
which were important for the assessment of DID. However, when mild DID was present with or without voluntary movements, their algorithm encountered difficulty in isolating dyskinesia. Yet, this represents an important step in the automatic detection and assessment of DID. Others have recently presented similar work using a combination of sEMG and accelerometers [47], a series of accelerometers [48], as well as gyroscopes and accelerometers [49] to assess DID, with results showing classification accuracy of $93 \%$ or better between dyskinesia and voluntary movements.

For most of the aforementioned studies, sensor position was determined based on the specific questions examined, and from educated guesses. Thus, the collected data provided information about one specific aspect of mobility, neglecting potential other key features that could be relevant. This is not a critic per se, in fact data reduction, i.e. reducing the number of sensors to a minimum to attain one's goal in capturing the desired mobility features, should be the ultimate goal. Nonetheless, restricting the amount of sensors may result in key information being missed. This is why it would be interesting to examine home mobility using a 'top-down' approach, i.e. have enough sensors to recreate whole-body movement, rather than a 'bottom-up' approach where the minimal number of sensors is used based on educated guesses. To date, there are only a few off-the-shelf systems that use multiple IMU sensors which comprise accelerometers, magnetometers and gyroscopes, e.g. [50]. These sensors are all linked into a biomechanical model that allows for 3D representation of whole-body movement within the natural environment. Usually, data from the sensors are streamed wirelessly to a receiving computer located within the recording space. Their accuracy and ability to perform long-term recordings are highly dependent on the quality of their inertial sensors; their built-in Kalman filters which help reduce noise within the system, as well as how the system can be modified in order to adapt it to the environment in which they are being used. Indeed, the integrity of the data will be highly dependent on how it behaves when magnetic disturbances are present due to the presence of magnetometers in the IMUs. The fast-pace development of such systems will most certainly allow for easier whole-body assessment, which can then be analyzed in detail to produce a series of key features (probably using mathematical means such as neural networks and others) and optimal number and location of sensors to capture the important aspects of movement needed to assess DID and its impact on the daily lives of patients with PD. To reach this goal, there will be several aspects to take into account (1) which key feature(s) better identify DID amongst voluntary movements, (2) how do those key features differ for different tasks, and (3) what is the optimal sensor placement to capture these key DID features.

Despite the obvious benefits, some important challenges remain. First, these types of sensor do not provide direct translational data. Only an estimation of DID is obtained from the fusion of the accelerometer, gyroscope and magnetometer data which is also limited by the algorithm used to perform this fusion. Second, there are currently limitations about distances traveled by patients. While some systems use data logging, others rely on wireless transmission of data to a computer located within the patient's home. Also, it is currently difficult to assess their true accuracy since no systematic comparison has been made with optical systems using a robust protocol that comprises multiple degrees of freedom and multiple sensors for long periods of time. Furthermore, most of the studies using these methods were performed on a limited number of patients. The high variability of motor disabilities within the PD population represents an obstacle for the development of automatic recognition and assessment of motor symptomatology. The differentiation of DID and tremor, such as for the laboratory assessment tools, is another challenge that needs to be addressed using the current natural environment assessment tools. Finally, the cost of such systems is still high, especially for those assessing whole-body movements. However, the fast pace development of these technologies, associated with a progressive reduction in cost, will eventually make such assessment methods available for all.

\section{CLINICAL EVALUATION VERSUS THE USE OF TECHNOLOGY}

Several methods described in the laboratory and home settings reported good correlations with clinical assessment. One could then come to the conclusion that clinical scales are as good as more elaborate technological methods, which can be more complicated to implement. However, this argument can be counteracted by the fact that long-term recording using multiple sensors could detect not only the magnitude of dyskinesia, but also its time-varying location on the body, and the time course of appearance of DID, with limited external intervention during recording. Furthermore, it could provide valuable information about strategies used by patients to counteract DID, with the development of advanced algorithms. Clini- 
cal scales, on the other hand, allow for the identification of specific types of DID, where more technologicallyoriented methods do not still possess the necessary sophistication to differentiate between the different types of dyskinesia. Nonetheless, one must keep in mind that the choice in assessment tool should always be guided by the specific question being asked.

\section{FUTURE CHALLENGES IN THE QUANTIFICATION OF DID}

Clinicians treating individuals with PD having DID face many challenges and difficult choices. First, they have to maintain optimal mobility of their patients, i.e. provide enough medication to avoid motor symptoms, while avoiding motor side effects such as DID. This task is rendered more difficult as the disease progresses. Once DID begins to occur, the clinician must then decide whether to modify medication dosage or to change the medication regimen by adding or withdrawing certain drugs. However, while dopamine agonists seem to reduce DID, they are not well tolerated by all patients, as they may increase psychiatric side effects [51-53]. The other possibility is simply to reduce the dosage of levodopa, but to the detriment of motor performance. The fact that bradykinesia is present with DID [38] seems to indicate that any reduction of levodopa has the potential to greatly reduce mobility. The solution may come with proper understanding of the impact of DID on voluntary motor acts. Indeed, the problem with actual treatment algorithms to manage DID is that they focus solely on whether DID is present or not, while putting aside how they affect everyday life. Does a reduction of $50 \%$ of DID in a particular point in time induce a $50 \%$ increase in functional mobility? In that particular case, it may be possible that the reduction in DID will be accompanied by an increase in bradykinetic features. On the other hand, a reduction of only $15 \%$ of DID may allow a patient to gain $40 \%$ of his or her functional capacities, as he or she is able to better control his or her hand during gross manipulation of objects without any major increase of bradykinetic symptoms. It may also be that the relationship between the amount of DID and mobility deficits is not linear. This 'signal-to-noise ratio' phenomenon, where the signal is the voluntary movement and the noise represents the DID, must be better understood before deciding whether the absence of DID is preferable. Furthermore, the understanding of the signal-to-noise ratio relationship between voluntary movements and DID would enable us to determine when DID becomes unmanageable for the patient. Indeed, patients must integrate the movement patterns of DID into the voluntary motor scheme in order to perform voluntary motor acts. In the signal-to-noise ratio rule, the difficulty in performing motor tasks will then be highly dependent on the amplitude of the voluntary act and the amplitude of DID in the limb performing the voluntary movement. Furthermore, the amount of close-loop (with sensory feedback) versus open-loop (movement performed with little or no sensory input) portion of movement will also dictate the influence of DID on performance. Finally, the velocity of the movement performed is also a key factor, as faster movements tend to increase the signal-to-noise ratio. Ideally, knowing the necessary signal-to-noise ratio for key daily activities, as well as better understanding how patients deal with DID during their everyday life could help us make great strides in deciding proper management of these motor fluctuations.

Of course, technological assessment of DID is not without limitations. First, there is the issue of availability; current instruments such as magnetic trackers, video-based motion capture system or inertial sensors are expensive, and they require substantial space to operate them, which is not readily available in a hospital setting. Second, they require highly-qualified technicians to operate them. This is why they are usually reserved for research purposes. Third, there are currently no commonly-accepted mathematical methodology to characterize DID, which in itself represents a challenge in interpreting the results. This is even evident for a simple evaluation of the amplitude of DID. Until there is consensus on how to examine characteristics of DID, it will be difficult to have them accepted as a "valid" and "reliable" method of quantifying DID. Fourth, DID possess a diurnal motor pattern which is described as an increase in the severity of DID later in the day $[23,54,55]$ due to a build-up of successive doses of levodopa/carbidopa [54]. Indeed, while a patient might present only with mild dystonia early in the morning, an assessment later in the day could identify DID. It is thus difficult to gather a complete profile of motor fluctuations experienced by a patient throughout the day; regardless of the assessment method used. Finally, there is the issue of acceptability by the clinical world. Clinicians are reticent to use such methods in their clinics because of the aforementioned reasons. The only way to circumvent the resistance by clinicians will be to (a) propose inexpensive, simple means to assess DID that require little or no qualified personnel, (b) such methods will have to provide information currently provided by clinical means of evaluation, 
but also information that is otherwise not available to clinicians. For example methodologies used to quantitatively assess DID in the natural environment of patients could help evaluate the effect of change of medication on DID amplitude and overall mobility during a week. This could provide clinicians with valuable information that is not necessarily available with simple clinical evaluation in the clinic. Nonetheless, much work remains to be done to provide such device to the clinical world.

\section{REFERENCES}

[1] Aubert I, Guigoni C, Hakansson K, Li Q, Dovero S, Barthe N, Bioulac BH, Gross CE, Fisone G, Bloch B, \& Bezard E (2005) Increased D1 dopamine receptor signaling in levodopainduced dyskinesia. Ann Neurol, 57(1), 17-26.

[2] Carta AR, Tronci E, Pinna A, \& Morelli M (2005) Different responsiveness of striatonigral and striatopallidal neurons to L-DOPA after a subchronic intermittent L-DOPA treatment. Eur J Neurosci, 21(5), 1196-1204.

[3] Cenci MA (2007) Dopamine dysregulation of movement control in L-DOPA-induced dyskinesia. Trends Neurosci, 30(5), 236-243.

[4] Gomez-Mancilla B, \& Bedard PJ (1992) Effect of chronic treatment with (+)-PHNO, a D2 agonist in MPTP-treated monkeys. Exp Neurol, 117(2), 185-188.

[5] Boraud T, Bezard E, Bioulac B, \& Gross CE (2001) Dopamine agonist-induced dyskinesias are correlated to both firing pattern and frequency alterations of pallidal neurones in the MPTP-treated monkey. Brain, 124(Pt 3), 546-557.

[6] Gordin A, Kaakkola S, \& Teravainen H (2004) Clinical advantages of COMT inhibition with entacapone - a review. J Neural Transm, 111(10-11), 1343-1363.

[7] Miller JW, Shukitt-Hale B, Villalobos-Molina R, Nadeau MR, Selhub J, \& Joseph JA (1997) Effect of L-Dopa and the catechol-O-methyltransferase inhibitor Ro 41-0960 on sulfur amino acid metabolites in rats. Clin Neuropharmacol, 20(1), 55-66.

[8] Mannisto PT, \& Kaakkola S (1999) Catechol-O-methyltransferase (COMT): Biochemistry, molecular biology, pharmacology, and clinical efficacy of the new selective COMT inhibitors. Pharmacol Rev, 51(4), 593-628.

[9] Fahn S (2000) The spectrum of levodopa-induced dyskinesias. Ann Neurol, 47(4 Suppl 1) p. S2-S9; discussion S9-S11.

[10] Klawans HL, Goetz C, \& Bergen D (1975) Levodopa-induced myoclonus. Arch Neurol, 32(5), 330-334.

[11] Melamed E (1979) Early-morning dystonia. A late side effect of long-term levodopa therapy in Parkinson's disease. Arch Neurol, 36(5), 308-310.

[12] Meunter MD, Sharpless NS, Tyce GM, \& Darley FL (1977) Patterns of dystonia (I-D-I and D-I-D) in response to Levodopa therapy of Parkinson's disease. Mayo Clinic Proceedings, 52(3), 163-174.

[13] Fahn, S., R.L. Elton (1987) and UPDRS program members, Unified Parkinsons Rating Scale, in Recent developments in Parkinsons disease, S. Fahn, et al., Editors. Macmillan Healthcare Information: Florham Park, p. 153-163.

[14] Goetz CG, Tilley BC, Shaftman SR, Stebbins GT, Fahn S, Martinez-Martin P, Poewe W, Sampaio C, Stern MB, Dodel R, Dubois B, Holloway R, Jankovic J, Kulisevsky J, Lang AE, Lees A, Leurgans S, LeWitt PA, Nyenhuis D, Olanow CW,
Rascol O, Schrag A, Teresi JA, van Hilten JJ, \& LaPelle N (2008) Movement Disorder Society-sponsored revision of the Unified Parkinson's Disease Rating Scale (MDS-UPDRS): scale presentation and clinimetric testing results. Mov Disord, 23(15), 2129-2170.

[15] Langston JW, Widner H, Goetz CG, Brooks D, Fahn S, Freeman T, \& Watts R (1992) Core assessment program for intracerebral transplantations (CAPIT). Mov Disord, 7(1), 213.

[16] Guy W (1976) Abnormal Involuntary Movement Scale, in ECDEU assessment manual for psychopharmacology, US Government Printing Office: Washington, DC, p. 534537.

[17] Goetz CG, Nutt JG, \& Stebbins GT (2008) The Unified Dyskinesia Rating Scale: Presentation and clinimetric profile. Mov Disord, 23(16), 2398-2403.

[18] Goetz CG, Stebbins GT, Shale HM, Lang AE, Chernik DA, Chmura TA, Ahlskog JE, \& Dorflinger EE (1994) Utility of an objective dyskinesia rating scale for Parkinson's disease: Inter- and intrarater reliability assessment. Mov Disord, 9(4), 390-394.

[19] Hagell P, \& Widner H (1999) Clinical rating of dyskinesias in Parkinson's disease: Use and reliability of a new rating scale. Mov Disord, 14(3), 448-455.

[20] Parkinson Study Group (2001) Evaluation of dyskinesias in a pilot, randomized, placebo-controlled trial of remacemide in advanced Parkinson disease. Arch Neurol, 58(10), p. 16601668 .

[21] Katzenschlager R, Schrag A, Evans A, Manson A, Carroll CB, Ottaviani D, Lees AJ, \& Hobart J (2007) Quantifying the impact of dyskinesias in PD: The PDYS-26: A patient-based outcome measure. Neurology, 69(6), 555-563.

[22] Colosimo C, Martinez-Martin P, Fabbrini G, Hauser RA, Merello M, Miyasaki J, Poewe W, Sampaio C, Rascol O, Stebbins GT, Schrag A, \& Goetz CG (2010) Task force report on scales to assess dyskinesia in Parkinson's disease: Critique and recommendations. Mov Disord, 25(9), 1131-1142.

[23] Nutt JG (2001) Motor fluctuations and dyskinesia in Parkinson's disease. Parkinsonism Relat Disord, 8(2), 101-108.

[24] Fenney A, Jog MS, \& Duval C (2008) Short-term variability in amplitude and motor topography of whole-body involuntary movements in Parkinson's disease dyskinesias and in Huntington's chorea. Clin Neurol Neurosurg, 110(2), 160-167.

[25] Goetz CG, Stebbins GT, Theeuwes A, Stocchi F, Ferreira JJ, van de Witte S, \& Bronzova J (2011) Temporal stability of the Unified Dyskinesia Rating Scale. Mov Disord.

[26] The Movement Disorders Society (2011); Available from: http://www.movementdisorders.org/publications/rating scales/.

[27] Yanagisawa N, \& Nezu A (1987) Pathophysiology of involuntary movements in Parkinson's disease. Eur Neurol, 26(Suppl 1), p. 30-40.

[28] Burkhard PR, Shale H, Langston JW, \& Tetrud JW (1999) Quantification of dyskinesia in Parkinson's disease: Validation of a novel instrumental method. Mov Disord, 14(5), 754-763.

[29] Duval C, Fenney A, \& Jog MS (2009) The dynamic relationship between voluntary and involuntary motor behaviors in patients with movement disorders, in Advances in Behavioral Biology, H.J. Groenewegen, et al., Editors. The Basal Ganglia IX, p. 521-534.

[30] Gour J, Edwards R, Lemieux S, Ghassemi M, Jog M, \& Duval C (2007) Movement patterns of peak-dose levodopa-induced dyskinesias in patients with Parkinson's disease. Brain Res Bull, 74(1-3), 66-74. 
[31] Liu X, Carroll CB, Wang SY, Zajicek J, \& Bain PG (2005) Quantifying drug-induced dyskinesias in the arms using digitised spiral-drawing tasks. J Neurosci Methods, 144(1), 47-52.

[32] Liu X, Osterbauer R, Aziz TZ, Miall RC, \& Stein JF (2001) Increased response to visual feedback of drug-induced dyskinetic movements in advanced Parkinson's disease. Neurosci Lett, 304(1-2), 25-28.

[33] Manson AJ, Brown P, O’Sullivan JD, Asselman P, Buckwell D, \& Lees AJ (2000) An ambulatory dyskinesia monitor. $J$ Neurol Neurosurg Psychiatry, 68(2), 196-201.

[34] Carroll CB, Bain PG, Teare L, Liu X, Joint C, Wroath C, Parkin SG, Fox P, Wright D, Hobart J, \& Zajicek JP (2004) Cannabis for dyskinesia in Parkinson disease: A randomized double-blind crossover study. Neurology, 63(7), 1245-1250.

[35] Rao AS, Bodenheimer RE, Davis TL, Li R, Voight C, \& Dawant BM (2008) Quantifying drug induced dyskinesia in Parkinson's disease patients using standardized videos. Conf Proc IEEE Eng Med Biol Soc, 2008, 1769-1772.

[36] Chung KA, Lobb BM, Nutt JG, McNames J, \& Horak F (2010) Objective measurement of dyskinesia in Parkinson's disease using a force plate. Mov Disord, 25(5), 602-608.

[37] Agostino R, Bagnato S, Dinapoli L, Modugno N, \& Berardelli A (2005) Neither simple nor sequential arm movements are bradykinetic in parkinsonian patients with peak-dose dyskinesias. Clin Neurophysiol, 116(9), 2077-2082.

[38] Ghassemi M, Lemieux S, Jog M, Edwards R, \& Duval C (2006) Bradykinesia in patients with Parkinson's disease having levodopa-induced dyskinesias. Brain Res Bull, 69(5), 512-518.

[39] Lemieux S, Ghassemi M, Jog M, Edwards R, \& Duval C (2007) The influence of levodopa-induced dyskinesias on manual tracking in patients with Parkinson's disease. Exp Brain Res, 176(3), 465-475.

[40] Fenney A, Jog MS, \& Duval C (2008) Bradykinesia is not a "systematic" feature of adult-onset Huntington's disease; implications for basal ganglia pathophysiology. Brain Res, 1193, 67-75.

[41] Mann RK, Edwards R, Zhou J, Jog M, \& Duval C (2010) Intraand inter-limb coherency during stance in non-dyskinetic and dyskinetic patients with Parkinson's disease. Clin Neurol Neurosurg, 112(5), 392-399.

[42] Chelaru MI, Duval C, \& Jog M (2010) Levodopa-induced dyskinesias detection based on the complexity of involuntary movements. J Neurosci Methods, 186(1), 81-89.

[43] Hoff JI, van den Plas AA, Wagemans EA, \& van Hilten JJ (2001) Accelerometric assessment of levodopa-induced dyskinesias in Parkinson's disease. Mov Disord, 16(1), 58-61.
[44] Hoff JI, van der Meer V, \& van Hilten JJ (2004) Accuracy of objective ambulatory accelerometry in detecting motor complications in patients with Parkinson disease. Clin Neuropharmacol, 27(2), 53-57.

[45] Keijsers NL, Horstink MW, van Hilten JJ, Hoff JI, \& Gielen CC (2000) Detection and assessment of the severity of levodopa-induced dyskinesia in patients with Parkinson's disease by neural networks. Mov Disord, 15(6), 11041111 .

[46] Keijsers NL, Horstink MW, \& Gielen SC (2003) Automatic assessment of levodopa-induced dyskinesias in daily life by neural networks. Mov Disord, 18(1), 70-80.

[47] Cole BT, Roy SH, De Luca CJ, \& Nawab S (2010) Dynamic neural network detection of tremor and dyskinesia from wearable sensor data. Conf Proc IEEE Eng Med Biol Soc, 2010, 6062-6065.

[48] Patel S, Lorincz K, Hughes R, Huggins N, Growdon J, Standaert D, Akay M, Dy J, Welsh M, \& Bonato P (2009) Monitoring motor fluctuations in patients with Parkinson's disease using wearable sensors. IEEE Trans Inf Technol Biomed, 13(6), 864-873.

[49] Tsipouras MG, Tzallas AT, Rigas G, Bougia P, Fotiadis DI, \& Konitsiotis S (2010) Automated Levodopa-induced dyskinesia assessment. Conf Proc IEEE Eng Med Biol Soc, 2010, 2411-2414.

[50] Schepers HM, Roetenberg D, \& Veltink PH (2010) Ambulatory human motion tracking by fusion of inertial and magnetic sensing with adaptive actuation. Med Biol Eng Comput, 48(1), 27-37.

[51] Avanzi M, Uber E, \& Bonfa F (2004) Pathological gambling in two patients on dopamine replacement therapy for Parkinson's disease. Neurol Sci, 25(2), 98-101.

[52] Lawrence AD, Evans AH, \& Lees AJ (2003) Compulsive use of dopamine replacement therapy in Parkinson's disease: Reward systems gone awry?. Lancet Neurol, 2(10), 595-604.

[53] Voon V (2004) Repetition, repetition, and repetition: Compulsive and punding behaviors in Parkinson's disease. Mov Disord, 19(4), 367-370.

[54] Joseph CL, Siple J, McWhorter K, \& Camicioli R (1995) Adverse reactions to controlled release levodopa/carbidopa in older persons: Case reports. J Am Geriatr Soc, , 43(1), 47-50.

[55] Merello M, Hughes A, Colosimo C, Hoffman M, Starkstein S, \& Leiguarda R (1997) Sleep benefit in Parkinson's disease. Mov Disord, 12(4), 506-508. 